\title{
Erratum: Improved First-Principles Calculation of the Third Virial Coefficient of Helium
}

\author{
Giovanni Garberoglio, ${ }^{1}$ Michael R. Moldover, ${ }^{2}$ and Allan H. Harvey ${ }^{3}$ \\ ${ }^{1}$ European Centre for Theoretical Studies in Nuclear Physics and Related Areas (FBK-ECT*) and \\ Trento Institute for Fundamental Physics and Applications (TIFPA-INFN), \\ Trento, I-38123 Italy \\ ${ }^{2}$ Sensor Science Division, National Institute of Standards and Technology, \\ Gaithersburg, MD 20899 \\ ${ }^{3}$ Applied Chemicals and Materials Division, National Institute of Standards and Technology, \\ Boulder, CO 80305 \\ garberoglio@ectstar.eu \\ allan.harvey@nist.gov
}

Key words: helium; virial coefficients.

Accepted: May 29, 2020

Published: June 6, 2020

https://doi.org/10.6028/jres.125.019

\section{Erratum}

The calculations of $C(T)$ for ${ }^{3} \mathrm{He}$ in the original paper [1] were in error at the lowest temperatures due to an incorrect accounting for the quantum statistics of fermions, as explained in Ref. [2]. A corrected version of Table 6 is given below. The calculations for ${ }^{4} \mathrm{He}$ are not affected by this error. 
Table 6. Third virial coefficients $C(T)$ for ${ }^{3} \mathrm{He}$ calculated in this work and our estimates (see Sec. 4.3) of their expanded $(k=2)$ uncertainties $U(C)$.

\begin{tabular}{|c|c|c|}
\hline $\begin{array}{l}T \\
\mathrm{~K}\end{array}$ & $\begin{array}{c}C \\
\mathrm{~cm}^{6} \cdot \mathrm{mol}^{-2}\end{array}$ & $\begin{array}{c}U(C) \\
\mathrm{cm}^{6} \cdot \mathrm{mol}^{-2}\end{array}$ \\
\hline 2.6 & 1659 & 55 \\
\hline 2.8 & 1648 & 42 \\
\hline 3 & 1605 & 36 \\
\hline 3.2 & 1560 & 32 \\
\hline 3.5 & 1473 & 26 \\
\hline 3.7 & 1403 & 24 \\
\hline 4 & 1321 & 20 \\
\hline 4.2 & 1274 & 17 \\
\hline 4.5 & 1185 & 15 \\
\hline 5 & 1075 & 11 \\
\hline 6 & 896.2 & 8.1 \\
\hline 7 & 775.9 & 5.9 \\
\hline 8.5 & 644.9 & 4.0 \\
\hline 10 & 553.7 & 3.0 \\
\hline 12 & 475.3 & 2.4 \\
\hline 13.8033 & 426.5 & 1.7 \\
\hline 15 & 401.8 & 1.6 \\
\hline 17 & 367.6 & 1.3 \\
\hline 18.689 & 346.9 & 1.0 \\
\hline 20 & 333.53 & 0.87 \\
\hline 24.5561 & 296.76 & 0.67 \\
\hline 30 & 268.91 & 0.52 \\
\hline 35 & 251.22 & 0.41 \\
\hline 50 & 218.56 & 0.26 \\
\hline 100 & 170.63 & 0.13 \\
\hline 150 & 146.71 & 0.10 \\
\hline 200 & 130.762 & 0.081 \\
\hline 273.16 & 114.365 & 0.068 \\
\hline 300 & 109.627 & 0.065 \\
\hline 400 & 95.707 & 0.058 \\
\hline 500 & 85.563 & 0.054 \\
\hline 750 & 68.758 & 0.049 \\
\hline 1000 & 58.168 & 0.047 \\
\hline 1500 & 45.083 & 0.045 \\
\hline 2000 & 37.130 & 0.044 \\
\hline
\end{tabular}

\section{References}

[1] Garberoglio G, Moldover MR, Harvey AH (2011) Improved first-principles calculation of the third virial coefficient of helium. Journal of Research of the National Institute of Standards and Technology 116:729-742. https://doi.org/10.6028/jres.116.016

[2] Garberoglio G, Harvey AH (2020) Erratum: Path-integral calculation of the third virial coefficient of quantum gases at low temperatures. Journal of Chemical Physics 152:199903. https://doi.org/10.1063/5.0010967

About the authors: Giovanni Garberoglio is a physicist at the European Centre for Theoretical Studies in Nuclear Physics and Related Areas (ECT*) in Trento, Italy. Michael Moldover is a physicist who is retired 
from the Sensor Science Division of the NIST Physical Measurement Laboratory. Allan Harvey is a chemical engineer in the Thermophysical Properties of Fluids Group in the Applied Chemicals and Materials Division of the NIST Material Measurement Laboratory. The National Institute of Standards and Technology is an agency of the U.S. Department of Commerce. 